\title{
Towards an End-to-end Radiation Defect Quantitative Characterization Workflow Using Advanced Microscopy Images
}

Rajat Sainju ${ }^{1}$, Graham Roberts ${ }^{2}$, Colin Ophus ${ }^{3}$, Brian Hutchinson ${ }^{4}$, Jing Wang ${ }^{4}$, Mychailo B. Toloczko ${ }^{4}$, Richard J Kurtz ${ }^{4}$, Charles H Henager Jr ${ }^{4}$, Danny J. Edwards ${ }^{4}$ and Yuanyuan Zhu ${ }^{1,4 *}$

${ }^{1}$ University of Connecticut, Storrs, Connecticut, United States, ${ }^{2}$ Lawrence Berkeley National Lab, Berkeley, California, United States, ${ }^{3}$ Western Washington University, Bellingham, Washington, United States, ${ }^{4}$ Pacific Northwest National Laboratory, Richland, Washington, United States

* Corresponding author: yuanyuan.2.zhu@uconn.edu

The safety and reliability of next-generation fission and future fusion energy systems depend in-part on the development of advanced reactor core structural materials. Changes in mechanical properties of nuclear reactor core structural materials under a radiation environment play a pivotal role in determining the structural integrity and lifetime of a reactor. To understand how radiation-induced extended defects govern the changes in alloy behavior, it is essential to characterize, and more importantly to quantify, these extended radiation defects. The current practice of defect analysis using transmission electron microscopy (TEM) images and deriving material property metrics such as dislocation density and precipitates/voids size distributions remains largely in the purview of human analysis. To date, there's no satisfactory solution to automate defect analysis for statistically meaningful quantification of a variety types of extended radiation defects in reactor core structural alloys.

In this work, we demonstrate a series of systematic efforts made to achieve a new characterization workflow for efficient and robust quantitative analysis of extended radiation defects in metallic alloys for nuclear energy applications, including commonly observed dislocation lines, dislocation loops, precipitates and voids. Figure 1 summaries this new end-to-end defect characterization workflow. To pave the way for reliable defect identification, we first established an advanced diffraction contrast imaging scanning transmission electron microscopy (DCI STEM) technique capable of recording defect contrast with high clarity, free of bend contour and other auxiliary artifacts [1]. Next, based on these high-quality defect images, we developed a novel deep convolutional neural network (CNN) architecture, called DefectSegNet [2], and trained it to facilitate defect semantic segmentation, i.e. it identifies which irradiation defects are present and precisely where they are located. Lastly, a set of dedicated MATLAB algorithms, called DefectQuant [3], were developed and tailored to extract quantitative information from each defect category with a full consideration of the matrix crystal structure, orientation and diffraction condition. All three steps of this new workflow were tested using a pair of pristine and neutron-irradiated HT-9 (a 12Cr tempered martensitic alloy) specimens neutron irradiated to $111.8 \mathrm{dpa}$ that contained a high density of extended defects. Easily accomplished, accurate, reproducible, and at least two orders of magnitude faster (than human experts) end-to-end defect quantification achieved here opens up new opportunities for readily obtaining statistically meaningful experimental data for understanding of radiation effects [4]. 


\section{Step 1: Defect Imaging : DCI-STEM}
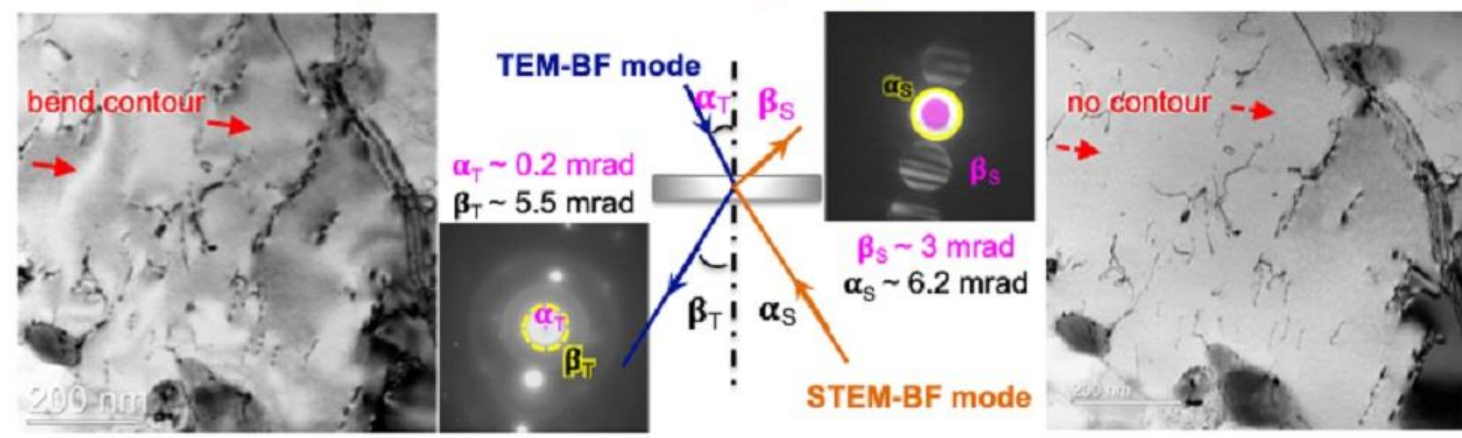

Step 2: Defect Reorganization:

Input DefectSegNet Deep Learning Algorithm Output mini-batch DefectSegNet Deep Learning Algorithm probability map $512 \times 512 \times 1 \quad 512 \times 512 \times 1$

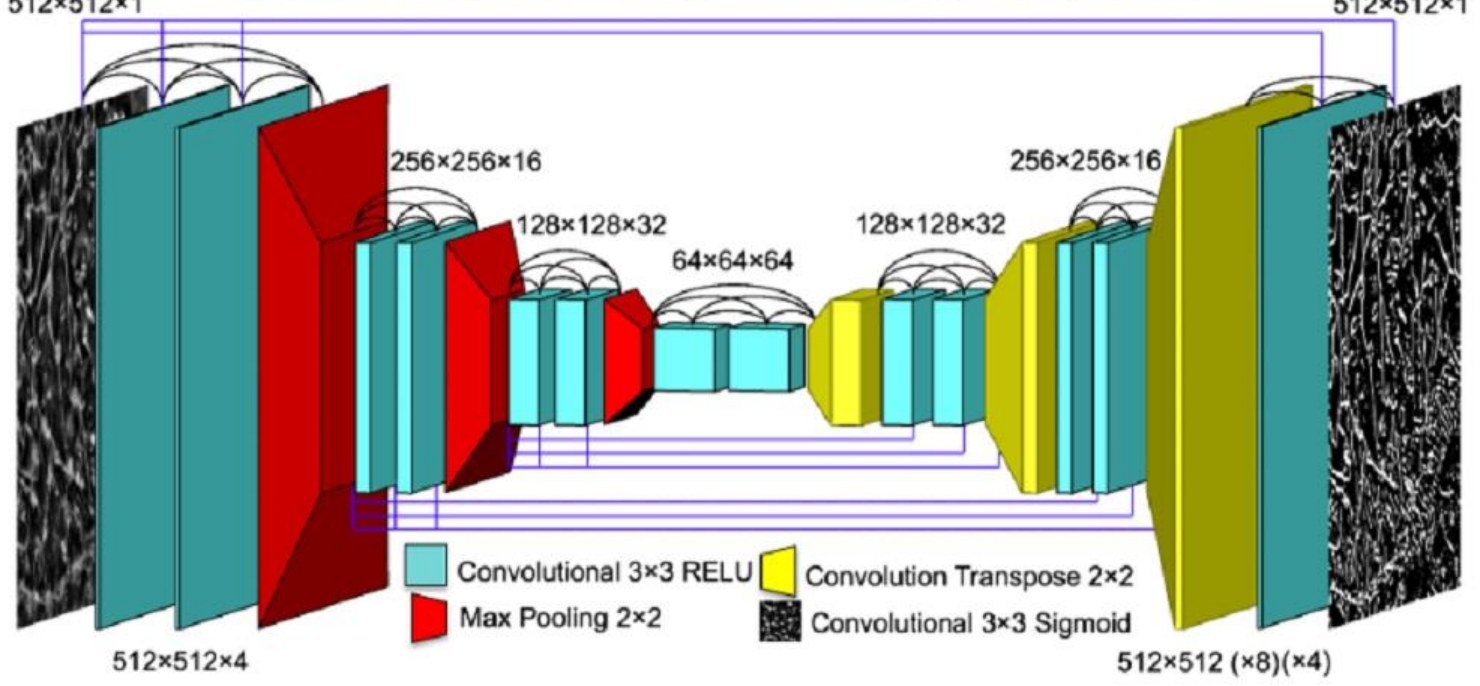

\section{Step 3: Defect Quantification: DefectQuant MATLAB Algorithm}

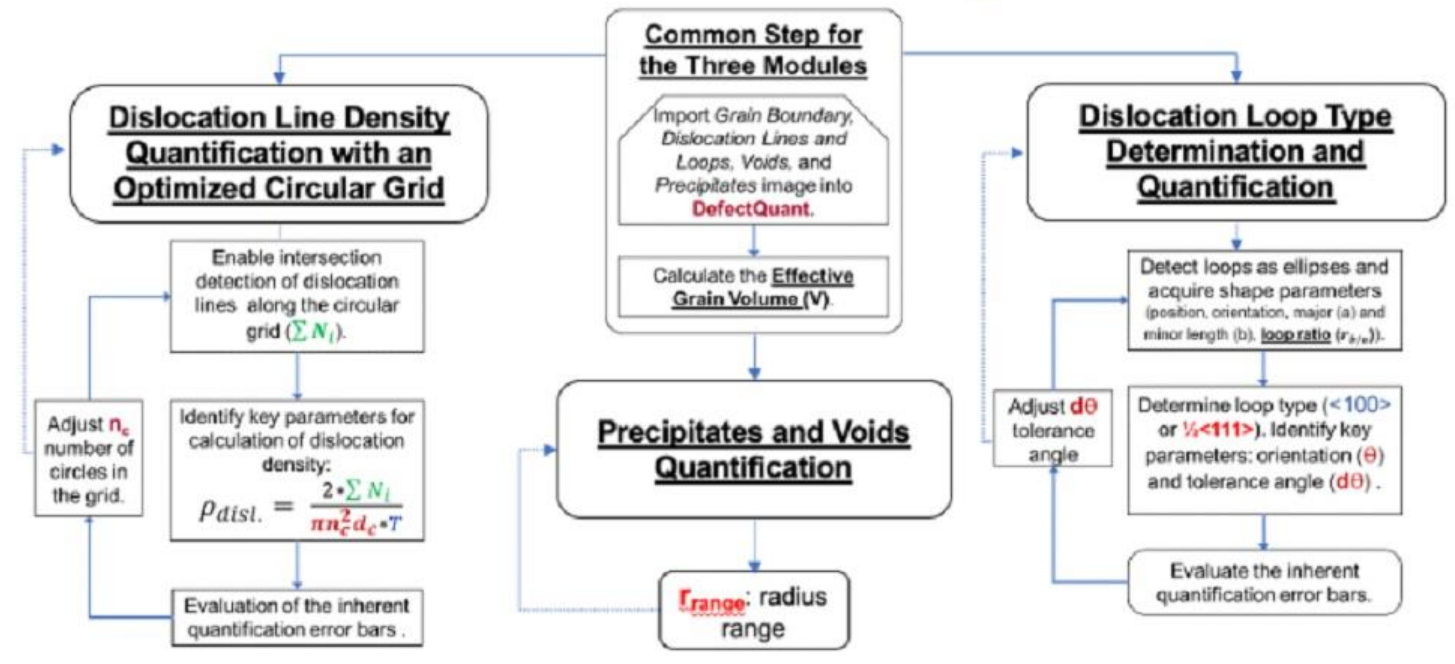


Figure 1. Schematic illustration of a new end-to-end radiation-induced extended defects characterization workflow consisting of defect imaging, reorganization and quantification.

\section{References}

[1] Y Zhu, C Ophus, MB Toloczko and DJ Edwards, Ultramicroscopy, 193(2018) 12-23.

[2] G Roberts, S Y Haile, R Sainju, D J Edwards, B Hutchinson and Y Zhu, Scientific Reports 9(2019), 12744

[3] R Sainju, C Ophus, MB Toloczko and DJ Edwards and Y Zhu. in preparation

[4] Authors thank Mr. Deep Patel for supporting image labeling. We acknowledge funding from the U.S. DOE Office of Fusion Energy Sciences under contract DE-AC05-76RL01830, Office of Nuclear Energy's Nuclear Energy Enabling Technologies program project CFA 16-10570, UConn HPC computing facility and PNNL Research Computing program for the GPUs used in this research. 